\title{
Extranatural Inflation
}

\section{Citation}

Arkani-Hamed, Nima, Hsin-Chia Cheng, Paolo Creminelli, and Lisa Randall. 2003. “Extranatural Inflation." Physical Review Letters 90 (22). https://doi.org/10.1103/physrevlett.90.221302.

\section{Permanent link}

http://nrs.harvard.edu/urn-3:HUL.InstRepos:41417286

\section{Terms of Use}

This article was downloaded from Harvard University's DASH repository, and is made available under the terms and conditions applicable to Other Posted Material, as set forth at http:// nrs.harvard.edu/urn-3:HUL.InstRepos:dash.current.terms-of-use\#LAA

\section{Share Your Story}

The Harvard community has made this article openly available.

Please share how this access benefits you. Submit a story.

Accessibility 


\title{
Extranatural Inflation
}

\author{
Nima Arkani-Hamed, Hsin-Chia Cheng, Paolo Creminelli and Lisa Randall \\ Jefferson Physical Laboratory, \\ Harvard University, Cambridge, MA 02138, USA
}

\begin{abstract}
We present a new model of inflation in which the inflaton is the extra component of a gauge field in a $5 \mathrm{~d}$ theory compactified on a circle. The chief merit of this model is that the potential comes only from non-local effects so that its flatness is not spoiled by higher dimensional operators or quantum gravity corrections. The model predicts a red spectrum $(n \simeq 0.96)$ and a significant production of gravitational waves $(r \simeq 0.11)$. We also comment on the relevance of this idea to quintessence.

PACS numbers: $98.80 . \mathrm{Cq}, 11.10 . \mathrm{Kk}$
\end{abstract}

Inflation is a theoretically attractive idea for solving many problems of Standard Big Bang cosmology, and recently many experiments have confirmed its predictions of a flat Universe with a nearly scale-invariant spectrum of adiabatic perturbations. However, despite its many successes, there are still no completely natural inflationary models known in particle physics. Although most of the literature concentrates on supersymmetric models, supersymmetry alone is not sufficient to protect the flatness of the inflaton potential. Supergravity corrections spoil the flatness because supersymmetry is broken during inflation [1]. The other candidate models for inflation rely on the inflaton taking values larger than the Planck mass, $M_{P}\left(M_{P} \equiv(8 \pi G)^{-1 / 2}\right)$. Examples of this possibility include chaotic inflation [2], and natural inflation [3, 4], to be discussed below. However, the use of a simple potential requires the fine-tuning of an infinite number of nonrenormalizable operators, suppressed by powers of $M_{P}$.

Natural inflation assumes that the inflaton is a Pseudo Nambu Goldstone Boson (PNGB), parametrized by an angular variable $\theta \sim \theta+2 \pi$. In the limit of exact symmetry, $\theta$ is an exactly flat direction. However, some tilt is necessary, and this is introduced by explicit symmetry breaking terms, resulting in a Lagrangian of the form

$$
\mathcal{L}=\frac{f^{2}}{2}(\partial \theta)^{2}-V_{0}(1-\cos (\theta)),
$$

where $f$ is the spontaneous breaking scale. The canonically normalized field is $\phi=f \theta$, so the potential is naturally a function of $\phi / f$, which can be flat for large $f$. This scenario is however problematic, because the requirements $\epsilon \ll 1$ and $\eta \ll 1$ on the slow-roll parameters

$$
\epsilon \equiv \frac{M_{P}^{2}}{2}\left(\frac{V^{\prime}}{V}\right)^{2} \sim \frac{M_{P}^{2}}{f^{2}}, \quad \eta \equiv M_{P}^{2} \frac{V^{\prime \prime}}{V} \sim \frac{M_{P}^{2}}{f^{2}}
$$

gives $f \gg M_{P}$. This implies the spontaneous breaking scale is above $M_{P}$ which is presumably outside the range of validity of an effective field theory description. Moreover, it is expected that quantum gravity effects, such as the virtual appearance of black holes, will explicitly break the global symmetry [5]. These effects, usually suppressed by powers of $f / M_{P}$, are here unsuppressed, so that it is hard to justify why $V_{0}$ should be smaller than $M_{P}$, as required by the COBE bound on the overall normalization of density perturbations: $\delta \rho / \rho \sim 10^{-5}$.

In this letter, we show that an extradimensional version of natural inflation can solve all the above problems, giving a very simple and trustworthy model of inflation.

Consider a $5 \mathrm{~d}$ model with the extra dimension compactified on a circle of radius $R$. The extra component $A_{5}$ of an abelian gauge field propagating in the bulk cannot have a local potential, due to the higher dimensional gauge invariance; a shift symmetry protects it similarly to what happens to a four-dimensional PNGB. A non-local potential as a function of the gauge invariant Wilson loop

$$
e^{i \theta}=e^{i \oint A_{5} d x^{5}}
$$

will however be generated in presence of charged fields in the bulk. For a non-abelian gauge group, also the self-interactions of the gauge bosons contribute to the potential. At energies below $1 / R, \theta$ is a $4 \mathrm{D}$ field with a Lagrangian of the form

$$
\mathcal{L}=\frac{1}{2 \cdot g_{4}^{2}(2 \pi R)^{2}}(\partial \theta)^{2}-V(\theta)+\cdots
$$

where $g_{4}^{2}=g_{5}^{2} /(2 \pi R)$ is the $4 \mathrm{D}$ gauge coupling, and the potential $V(\theta)$ is given at one-loop by $6,6,8,9,10$

$$
V(\theta)=-\frac{1}{R^{4}} \sum_{I}(-1)^{F_{I}} \frac{3}{64 \pi^{6}} \sum_{n=1}^{\infty} \frac{\cos (n q \theta)}{n^{5}}
$$

where $F_{I}=0(1)$ for massless bosonic (fermionic) fields of charge $q$ coupled to $A_{5}$. Note that the potential is of the same form as in natural inflation (with small corrections from additional terms in the sum), with the effective decay constant given by

$$
f_{\text {eff }}=\frac{1}{2 \pi g_{4 \mathrm{~d}} R} .
$$

It is easily seen that $f_{\text {eff }}$ can be bigger than $M_{P}$ for sufficiently small $g_{4 \mathrm{~d}}$; the slow-roll condition $f_{\text {eff }} \gg M_{P}$ 
requires only that

$$
2 \pi g_{4 \mathrm{~d}} M_{P} R \ll 1
$$

The canonically normalized field is $\phi=\theta f_{\text {eff }}$. Due to the higher dimensional nature of the model, the potential (5) can be trusted even when the $4 \mathrm{~d}$ field $\phi$ takes values above $M_{P}$; no dangerous higher-dimension operator can be generated in a local higher-dimensional theory. This conclusion is quite important as it is commonly believed that any inflation model with field values above $M_{P}$ cannot be justified from a particle physics point of view; we see that this conclusion is valid only if we restrict to purely $4 \mathrm{~d}$ models. Quantum gravity corrections to the potential (5) are negligible if the extra dimension is bigger than the Planck length, different from what is expected in a 4d PNGB model. Again locality in the extra space is the key feature; virtual black holes cannot spoil the gauge invariance and do not introduce a local potential for $A_{5}$, while non-local effects are exponentially suppressed by $\sim e^{-2 \pi M_{5} R}$, because the typical length scale of quantum gravity effects (the $5 \mathrm{~d}$ Planck length $M_{5}^{-1}$ ) is much smaller than the size of the extra dimension.

It is now clear that $\phi$ is a good candidate for the inflaton. The extra-dimensional nature of the model has no cosmological consequences aside from constraining the quantum corrections to the inflaton potential, assuming the extra dimension is stabilized. Moreover, one can check that the Hubble length $H^{-1}$ is much larger than the size of the extra dimension so that the theory can be treated as 4-dimensional during inflation.

To keep quantum gravity corrections under control we can estimate a lower bound on the size of the extra dimension

$$
R \gtrsim 5 \cdot M_{P}^{-1}
$$

From this inequality the slow-roll condition (17) requires

$$
g_{4 \mathrm{~d}} \lesssim 10^{-2}
$$

this is equivalent to saying the dimensionful $5 \mathrm{~d}$ gauge coupling satisfies

$$
\frac{1}{g_{5 \mathrm{~d}}^{2}} \gtrsim 10^{3} M_{5}
$$

An upper bound on the size of the extra dimension can be obtained using the COBE normalization for the curvature density. The maximum value of $R$ will be obtained inflating near the top of the potential so that the vacuum energy is given by

$$
V_{0} \sim \frac{c}{16 \pi^{6}} \frac{1}{R^{4}}, \quad c \sim \mathcal{O}(1)
$$

where $c$ depends on the number of charged fields in the bulk. In this case the curvature density can be estimated by

$$
\delta_{H}=\frac{1}{5 \sqrt{3} \pi} \frac{V^{3 / 2}}{M_{P}^{3} V^{\prime}} \sim \frac{1}{20 \sqrt{6} \pi^{4}}\left(\frac{c}{M_{P}^{4} R^{4} \epsilon}\right)^{1 / 2} .
$$

The constraint of the COBE measurement

$$
\delta_{H}=1.91 \times 10^{-5},
$$

implies the size of extra dimension, $R$, to satisfy

$$
R \lesssim c^{1 / 4} \cdot\left(10^{17} \mathrm{GeV}\right)^{-1}
$$

where the equality is reached taking $\epsilon$ as big as presently allowed by constraints on $n:|n-1| \lesssim 0.1$. Note that the extra dimension is very small and it can be stabilized by a generic mechanism without affecting the cosmological evolution up to very high scales.

As a consequence of the smallness of the slow-roll parameters and of the density perturbations, a small parameter seems quite unavoidable in any model of inflation: this is the case for the gauge coupling (9) in our model. Nevertheless, note that the limit $g_{5} \rightarrow 0$ is natural in the 't Hooft sense. For $g_{5}=0$ we have a U(1) gauge symmetry with no charged field; this symmetry forbids gravity to make $g_{5} \neq 0$ and a similar reasoning holds for non-abelian groups.

For $\left(2 \pi g_{4 d} R\right)^{-1} \gg M_{P}$, the potential can be expanded in powers of $\phi$ and the density perturbations in the observable window is determined by the lowest order term, the mass term. The predictions are then the same as those of the chaotic inflation model with a parabolic potential [2]. The spectral index is given by $n=1-2 / N_{\mathrm{COBE}}$, where $N_{\mathrm{COBE}}$ is the number of e-folds between the exit of wavelengths now comparable to the observable Universe and the end of inflation. The reheating temperature is determined by the $A_{5}$ decay through gauge interactions. This gives $T_{\mathrm{rh}} \sim 10^{13}-10^{14} \mathrm{GeV}$, when $R$ and $g_{4 \mathrm{~d}}$ saturate the bounds (8) and (9) [11] and it scales as $g_{4 \mathrm{~d}}^{3 / 2} R^{-1 / 2}$. $N_{\mathrm{COBE}}$ can be estimated to lie in the interval $55-60$. The spectrum is therefore red-tilted:

$$
n \simeq 0.96
$$

a value not far from the present experimental sensitivity and compatible with the recent WMAP data 12].

It is known that a significant gravitational wave contribution requires large enough vacuum energy during inflation. Combined with the slow-roll conditions and the COBE normalization this requires a variation of the inflaton field bigger than the Planck scale [13], which again typically appears to be out of control of the effective theory description. As we stressed, this conclusion holds only for $4 \mathrm{~d}$ scenarios, while our model predicts a relative amplitude of the gravitational waves and density perturbations 14]

$$
r \simeq 12.4 \epsilon=6.2 / N_{\mathrm{COBE}} \sim 0.11
$$


which could be detected by the forthcoming Planck satellite.

For $\left(2 \pi g_{4 d} R\right)^{-1}$ close to $M_{P}$, higher power terms are non-negligible and the predictions will deviate from those based on the simple monomial potential; the spectral index becomes in this case even smaller than (15) 15] and therefore would be at least as measurable. On the contrary the contribution of gravity waves becomes smaller and difficult to measure for small $f_{\text {eff }}[4]$.

One could ask whether it is possible to derive a purely $4 \mathrm{~d}$ theory by the recent idea of deconstructing dimensions, where the Wilson line in the extra dimension corresponds to a 4d PNGB 16, 17, 18, 19. In this case one replaces the $5 \mathrm{~d}$ gauge theory by a (closed) chain of $4 \mathrm{~d}$ gauge groups, with the adjacent gauge groups connected by the link fields, which get nonzero VEVs and break the gauge groups down to the diagonal one. There is one linear combination of the Nambu-Goldstone bosons not eaten by the massive gauge fields. It remains light and corresponds to the non-local Wilson line field in the $5 \mathrm{~d}$ case. However the required symmetry breaking scale,

$$
f_{\text {eff }}=\frac{f_{\text {link }}}{\sqrt{N}}
$$

where $f_{\text {link }}$ is the $\mathrm{VEV}$ of the link fields and $N$ is the number of the sites, requires the problematic relation $f_{\text {link }} \gg M_{P}$. The point is that although we can simulate the locality in extra dimensions in the gauge sector by deconstruction, we did not modify the nature of the $4 \mathrm{~d}$ gravity which is the origin of the problems [20]. Purely $4 \mathrm{~d}$ models based on a PNGB with $f \ll M_{P}$ can however be constructed, though they involve more structure than the simple extra-dimensional scenario we have described 22 .

Recent observations indicate that most of the energy of the Universe is given by a negative pressure component. A candidate for this component is a nearly massless, slow-rolling scalar, called the quintessence field. The extreme flatness of the potential and the bounds coming from the absence of long range forces mediated by this scalar indicate that the quintessence field could be a PNGB 23, 24]. The problems of this proposal include those of the 'natural inflation' scenario because the spontaneous breaking scale is again required to be comparable or bigger than $M_{P}$ 21]. Again using the extra component of a gauge field as quintessence, one can avoid this problem and obtain a naturally flat potential. Still, the required flatness demands very small parameters: the quintessence mass must be smaller than the present Hubble scale $m \sim g_{4 \mathrm{~d}} / R \lesssim H_{0} \simeq 10^{-33} \mathrm{eV}$. Either a very small gauge coupling or a very large extra dimension is required, in the absence of other model-building ideas.

It is interesting to ask whether the slow-roll condition $g_{4 \mathrm{~d}} R M_{P} \ll 1$ can naturally arise in string compactifications containing a circle around which we can wrap a Wilson line $e^{i \theta}$. For instance if the gauge group lives in ten dimensions and spacetime is compactified to four dimensions, then in type I theory $g_{4 \mathrm{~d}}^{2} \sim g_{s} /\left(V_{6} M_{s}^{6}\right)$ and $M_{P}^{2} \sim g_{s}^{-2} M_{s}^{8} V_{6}$, so that $g_{4 \mathrm{~d}} M_{P} R \sim g_{s}^{-1 / 2} R M_{s}$. For this to be much smaller than one in the perturbative regime, we then require the radius $R \ll M_{s}^{-1}$, much smaller than the string length, which is not in the regime of validity of our effective field theory description. Nevertheless we can take the $T$ dual to get a convenient description of the physics. The $T$ dual theory has a radius $\tilde{R}=1 /\left(M_{s}^{2} R\right) \gg M_{s}^{-1}$, and the Wilson line for a nonabelian group turns into an angle $\theta$ between D-branes on the circle. Using the distance between branes as an inflaton is the idea of brane inflation [25]. Note however that in this regime the potential between the branes is naturally a function of the distance $\tilde{R} \theta$ between them, so on dimensional grounds the potential is of the form $V(\theta) \sim M_{s}^{4} F\left(\tilde{R} \theta M_{s}\right)$. Since $\tilde{R} M_{s} \gg 1$, the largeness of the effective $f$ does not in itself guarantee that the potential is sufficiently flat. Finding stringy scenarios that naturally lead to $g_{4 \mathrm{~d}} M_{P} R \ll 1$ while leading to the effective 5-dimensional field theory regime we have been using then remains an important and interesting challenge [26].

In conclusion we have shown that the extra component of a gauge field in a 5d theory is a good candidate for the inflaton. The predictions of our model are similar to the chaotic model with parabolic potential, so that our proposal can be considered a sensible particle physics embedding of this simple scenario. Locality in the extra dimension protects the flatness of the potential against Planck scale effects, even if the inflaton takes values above the Planck scale. As there is no trustworthy model in $4 \mathrm{~d}$ with a variation of the inflaton field bigger than $M_{P}$, the detection of a gravitational wave contribution to the CMBR anisotropy, would probably point to the existence of extra dimensions or other modifications of $4 \mathrm{~d}$ gravity.

[1] E. J. Copeland, A. R. Liddle, D. H. Lyth, E. D. Stewart and D. Wands, Phys. Rev. D 49, 6410 (1994) astro-ph/9401011.

[2] A. D. Linde, Phys. Lett. B 129, 177 (1983).

[3] K. Freese, J. A. Frieman and A. V. Olinto, Phys. Rev. Lett. 65, 3233 (1990).

[4] F. C. Adams, J. R. Bond, K. Freese, J. A. Frieman and A. V. Olinto, Phys. Rev. D 47, 426 (1993) hep-ph/9207245.

[5] R. Kallosh, A. D. Linde, D. A. Linde and L. Susskind, Phys. Rev. D 52, 912 (1995) hep-th/9502069.

[6] Y. Hosotani, Phys. Lett. B 126, 309 (1983).

[7] H. Hatanaka, T. Inami and C. S. Lim, Mod. Phys. Lett. A 13, 2601 (1998) hep-th/9805067.

[8] I. Antoniadis, K. Benakli and M. Quiros, New J. Phys. 3, 20 (2001) hep-th/0108005.

[9] G. von Gersdorff, N. Irges and M. Quiros, Nucl. Phys. B 635, 127 (2002) hep-th/0204223. 
[10] H.-C. Cheng, K. T. Matchev and M. Schmaltz, Phys. Rev. D 66, 036005 (2002) hep-ph/0204342.

[11] The natural inflation scenario predicts a much lower reheating temperature, $T_{\mathrm{rh}} \lesssim 10^{8} \mathrm{GeV}[3]$. In fact, in the extra-dimensional case, the potential for $A_{5}$ is protected even in presence of unsuppresed couplings with other particles, due to gauge invariance and locality. A 4d PNGB has only derivative interactions in the limit of exact symmetry, so that its decay rate is suppressed.

[12] WMAP collaboration, astro-ph/0302225

[13] D. H. Lyth, Phys. Rev. Lett. 78, 1861 (1997) hep-ph/9606387.

[14] For the definition of $r$ and a comprehensive review on inflation see A. R. Liddle and D. H. Lyth, "Cosmological Inflation And Large-Scale Structure," Cambridge, UK: Univ. Pr. (2000) $400 \mathrm{p}$.

[15] T. Moroi and T. Takahashi, Phys. Lett. B 503, 376 (2001) hep-ph/0010197.

[16] N. Arkani-Hamed, A. G. Cohen and H. Georgi, Phys. Rev. Lett. 86, 4757 (2001) hep-th/0104005.

[17] C. T. Hill, S. Pokorski and J. Wang, Phys. Rev. D 64, 105005 (2001) hep-th/0104035.
[18] H.-C. Cheng, C. T. Hill, S. Pokorski and J. Wang, Phys. Rev. D 64, 065007 (2001) hep-th/0104179.

[19] N. Arkani-Hamed, A. G. Cohen and H. Georgi, Phys. Lett. B 513, 232 (2001) hep-ph/0105239.

[20] In the opposite case $f \ll M_{P}$ (e.g. for the axion) a deconstructed extra-dimension suppresses quantum gravity corrections; for gauge invariance they must contain all the link fields, so that they are suppressed by $\left(f / M_{P}\right)^{N}$, where $N$ is the number of sites [21].

[21] C. T. Hill and A. K. Leibovich, Phys. Rev. D 66, 075010 (2002) hep-ph/0205237.

[22] N. Arkani-Hamed, H. C. Cheng, P. Creminelli and L. Randall, hep-th/0302034

[23] J. A. Frieman, C. T. Hill, A. Stebbins and I. Waga, Phys. Rev. Lett. 75, 2077 (1995) astro-ph/9505060.

[24] S. M. Carroll, Phys. Rev. Lett. 81, 3067 (1998) astro-ph/9806099.

[25] G. R. Dvali and S. H. Tye, Phys. Lett. B 450, 72 (1999) hep-ph/9812483.

[26] T. Banks, M. Dine, P. J. Fox and E. Gorbatov, hep-th/0303252 